\title{
COVID-19 and Computation for Policy
}

\author{
by Jeffrey Johnson, Peter Denning, Kemal Delic, and Jane Bromley
}

\begin{abstract}
Editor's Introduction
Governments across the world are formulating and implementing medical, social, economic and other policies to manage the COVID-19 pandemic and protect their citizens. Many governments claim that their policies follow the best available scientific advice. Much of that advice comes from computational modeling. Two of the main types of model are presented: the SIR (Susceptible, Infected, Recovered) model developed by Kermack and McKendrick in the 1920 s and the more recent Agent Based Models. The SIR model gives a good intuition of how epidemics spread; including how mass vaccination can contain them. It is less useful than Agent Based Modeling for investigating the effects of policies such as social distancing, self-isolation, wearing facemasks, and test-trace-isolate.
\end{abstract}

Politicians and the public have been perplexed to observe the lack of consensus in the scientific community and there being no single 'best science' to follow. The outcome of computational models depends on the assumptions made and the data used. Different assumptions will lead to different computational outcomes, especially when the available data are so poor. This leads some commentators to argue that the models are wrong and dangerous. Some may be, but computational modeling is one of the few ways available to explore and try to understand the space of possible futures. This lack of certainty means that computational modeling must be seen as just one of many inputs into the political decision making process. Politicians must balance all the competing inputs and make timely decisions based on their conclusions-be they right or wrong. In the same way that democracy is the least worst form of government, computational modeling may be the least worst way of trying to understand the future for policy making. 


\title{
COVID-19 and Computation for Policy
}

\author{
by Jeffrey Johnson, Peter Denning, Kemal Delic, and Jane Bromley
}

Worldwide the COVID-19 pandemic has been one of the greatest medical, social, economic, and political shocks the world has experienced since the Second World War. Economies around the world are reeling and taking on unprecedented levels of debt as they to try to survive the pandemic. Worldwide, governments are adopting policies, which they say, "follow the science." That science includes traditional sciences such as biology, medicine, and epidemiology combined with the approach of complex systems science and computational modeling. We explain this new computer enabled science and how it is used to create computational models that enable policy makers to forecast possible futures and possible outcomes of policy as they try to manage this multi-trillion dollar global catastrophe. We also examine some of the controversies associated with modeling and policy.

\section{The Emergence of a Deadly New Virus}

In the autumn of 2019 a new virus appeared in China. The world watched on as the epidemic spread alarmingly through the eleven million people of the city of Wuhan in Central China's Hubei province. China's new "flu" seemed far away in December 2019. Most countries around the world did not see the significance of this until January 2020 and even then the scale of the threat was not fully appreciated until two months later. Some countries were following their experience with SARS and Ebola, where local containment had proved successful. COVID did not follow the same pattern, taking everyone by surprise. For example, on January 24, 2020 Public Health England said the risk was low; by January $31^{\text {st }}$ this was raised to the risk being medium; by the $4^{\text {th }}$ of March the British government issued instructions on hand washing and social distancing by two meters in public places; and by March $26^{\text {th }}$ it declared a hugely expensive national lockdown policy in response to research from Imperial College London [1].

The spread of a new coronavirus, designated COVID-19 by the World Health Organization (WHO), was declared on March 11, 2020 to be a pandemic: "Pandemic is not a word to use lightly or carelessly. It is a word that, if misused, can cause unreasonable fear, or unjustified acceptance that the fight is over, leading to unnecessary suffering and death. This is the first pandemic caused by a coronavirus and we have never before seen a pandemic that can be controlled ... we have called every day for countries to take urgent and aggressive action. ... We have rung the alarm bell loud and clear." [2]. "COVID-19 is transmitted primarily by particles that can travel two meters or more when an infected person coughs or sneezes and contamination of hard surface where the virus can survive for a few hours" [3]. The infection transmission depends on number of viral particles multiplied by time of contact. Passing 
someone wearing a mask in a socially distanced store in a few seconds is less risky than socializing in a noisy crowded pub. Frontline workers in the health system are particularly vulnerable. In Europe the most severe early cases of the pandemic occurred in Northern Italy where the health system was overwhelmed with severely ill people who could not all be treated in hospital [4].

COVID-19 is a new virus and in January 2020 almost nothing was known about it. In the months that followed policy makers were faced with the completely new problem of estimating how many people would be infected, how fast the virus would spread, how many acute hospital beds would be required, how much PPE (personal protective equipment) would be required, how many testing kits would be required, and so on. To answer these urgent questions they turned to their scientists and computational modelers.

\section{Policy Following the Science}

In the early days of the pandemic politicians in many countries repeatedly said that they were "following the science." For example, on March 11, 2020 the U.K. Minister of Health said in the House of Commons, "Our approach will be guided by the best scientific evidence and medical advice, and we will take all necessary measures to deal with this outbreak" [5]. Most countries have scientific laboratories and university departments with expert knowledge on epidemics. In the U.K. the government appeals to its Scientific Advisory Group for Emergencies (SAGE) to advise it on COVID-19. However what appears to be a sensible partnership between objective scientists sharing scientific facts and politicians deciding the best course of action ignores the essential nature of scientific knowledge as contingent and contested. As time went by it became clear there were disagreements on the science between members of SAGE and other scientists, and even scientific disagreements within SAGE. In May 2020 a former government chief scientific advisor set up an "alternative SAGE" [6]. As it became clear that there was not a single definitive science to follow, political decisions had to be made which, if they went wrong, could not be blamed on "the science." It is the nature of science that its progress depends on fierce battles between alternative theories and viewpoints. Although there is great consensus across vast swathes of science, the scientific community rarely has a single view on anything new.

\section{Lockdown}

Sooner or later governments worldwide imposed different variants of "lockdown" requiring people to stay at home isolating themselves, with most business closed and many people being "furloughed." In the U.K. this meant people receiving a reduced government-funded income from their employer instead of being let go so that the business could start again when the epidemic was over. Even so many people were laid off and many furloughed people will still lose their jobs as businesses adjust to the new economic situation after lockdown. For example 
on July $1^{\text {st }} 12,000$ U.K. job losses were announced including many from iconic department stores such John Lewis and Harrods [7]. In Britain the government has committed a budget of billions of pounds to keep the economy on life support until it has recovered. The social and economic cost of lockdown is enormous. Also lockdown has increased the number of nonCOVID deaths including untreated cancers and suicides.

\section{Policy Needs Forecasts of the Future}

Policy makers need to know what might happen next, and what will be the likely outcomes of their policies. The conventional scientific approach cannot answer these questions. Pandemics are complex, highly dynamic multilevel systems. They do not respect traditional discipline boundaries between the biological, medical, social, economic, and other sciences. Global spread and infection numbers emerge at the macro level from the micro-level activities and interactions of people in a very wide variety of individual social situations.

Most systems administered by politicians cannot be predicted in a conventional scientific sense. Prediction is the gold standard of conventional science. Theories live or die by their ability to predict events in the future and by experiments showing the predictions are correct. Generally these are point predictions that a particular event will occur at a particular point in time. Such predictions are rarely possible in complex systems. There are many interrelated reasons for this but together they make the dynamics of complex multilevel systems "sensitive to initial conditions." Change the starting position slightly at any level and the outcome can be very different. For example, in the U.K. the apparently minor decision not to maintain a complete stock of protective personal equipment (PPE) in 2009 contributed greatly to the inability to protect frontline medical and service staff in 2020 [8]. Complex systems cannot be predicted in a deterministic sense. At best future events can be identified and approximate estimates made of their likelihood. Apart from guesswork and blind certainty, the only scientific way to forecast possible behaviors of complex systems is mathematical, statistical, and computer modeling and computer simulation building on conventional scientific knowledge.

\section{Models and Computation}

A model is a description of a system or process to show how it works. We have mental models about all aspects of our lives and we use them to try to understand things and decide what to do. The idea of a scientific model is that the "real world" is mapped to an abstraction in words, numbers, pictures, etc. Within that abstraction there are transition rules that map the model from its state "now" to another state in future time (Figure 1). In traditional science if the modeled dynamics and the real dynamics give the same outcome, the modeled dynamics are accepted as a viable theory of the actual dynamics. This validation has to be repeated many times for people to trust it. 


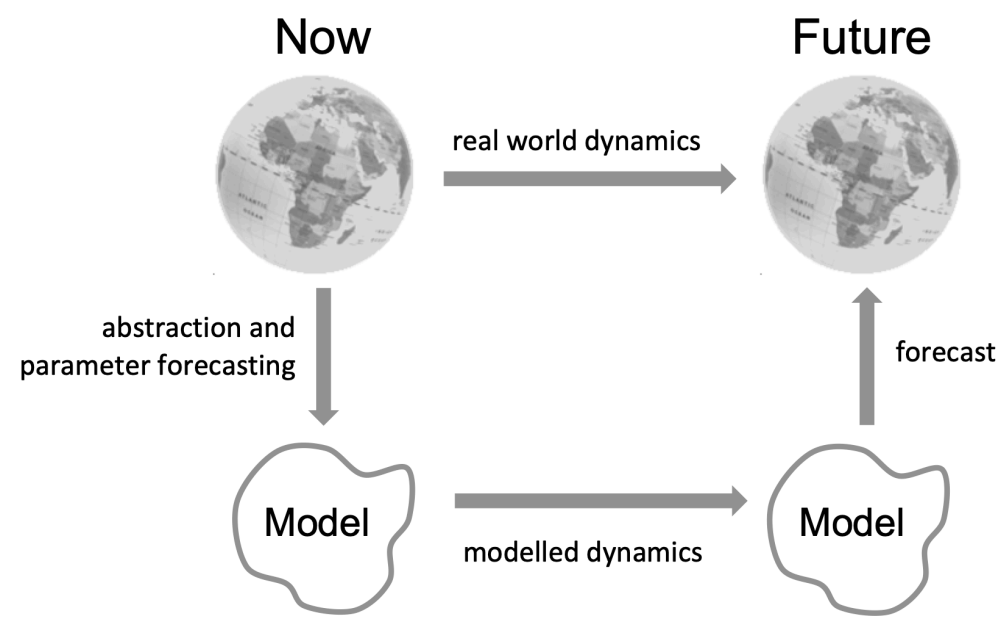

Figure 1. A simplified view of modeling.

Typically modeling proceeds in two stages: (i) The model is compared with reality to see how well it predicts outcomes when the only information provided is parameter measurements from the actual world. Then (ii) forecasting is done with a validated model where the main uncertainty is the values of the parameters in the future time period. The modeler has to make assumptions about those future parameter values, which then go into the model to yield the forecast. Most science aims to work with validated models and validated methods of parameter forecasting, and the best science makes clear the underlying assumptions.

With complex systems the modeled dynamics are not expected to produce point predictions. In fact the science of complex systems allows-or expects-the modeled dynamics to produce different forecasts of future modeled states. A further complication is that complex systems may undergo local or global phase transitions so assumptions that are valid at one time become invalid at another [9]. Indeed one of the defining features of complex adaptive systems is that they can reconfigure themselves and behave in new and different ways [10].

Complex systems science is computer enabled and one of its most powerful tools is simulation by iterated computation. This involves computing the system state at the next tick of the clock from its current state using transition rules. For systems that are sensitive to initial conditions this kind of computer modeling involves running simulation programs many thousands or millions of times to produce distributions of outcomes. This gives a view of the space of future events rather than saying that any particular event will occur. A single run of a computer simulation tells you almost nothing. Many runs from many starting positions are needed to populate the space of future possibilities so that its shape and the likelihood of alternative events can be evaluated. For these reasons real-world computer modeling often requires large powerful computational infrastructure. Nonetheless useful computer modeling can be done on standard PCs and notebook computers. Also modeling embraces Al, loT, and social media, e.g., the new apps being developed using mobile telephony to collect contact information to support the test-trace-isolate policies used to try to contain the pandemic at local levels. 


\section{Macro-level Epidemic Models}

A key parameter for epidemics is the basic reproduction number, $R_{0}$, which is defined to be the number of people an infected person will infect at the beginning of an epidemic. This depends on many things, some biological and some cultural, such as shaking hands, kissing, hugging, and close contact at shared meals, parties, and musical events. As an epidemic develops the reproduction number, $R$, may change according to the number of susceptible people, local circumstances, and policy interventions.

In 1927 Kermack and McKendrick proposed one of the first models of epidemics [11]. It was called the SIR model and could be used to investigate the relationships between the numbers of susceptible (S), infected (I), and recovered (R) people in a population. Interest in the SIR model was rekindled by a paper by Anderson and May in 1992 [12]. The model's abstraction is that there is a population made up of susceptible people, infected people, and recovered people. In the basic SIR model it is assumed that when people have recovered they are not susceptible to reinfection. In the SIR model the reproduction number $R$ is defined as the infection rate divided by the recovery rate. When the infection rate is greater than the recovery rate $(R>1.0)$ an epidemic can occur. When the recovery rate is greater than the infection rate $(R<1.0)$ the epidemic dies out. This model is based on differential equations that link the increases and decreases in the numbers of susceptible people (blue curve), infected (red), and recovered (green) as shown in Figure 2.

The basic SIR model (https://cs-dc.uk/sir.html) is easy to program and gives results similar to those in Figure 2(a). The red curve shows the proportion of the population infected at any time. The worry for policy makers is that the peak of this red curve for infected people may swamp the available healthcare provision, as was seen in Italy in March 2020 [4].

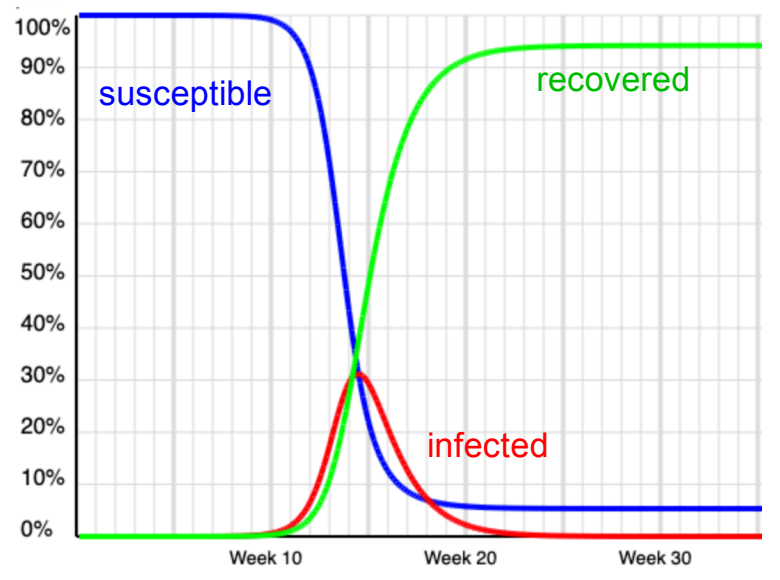

(a) The SIR model

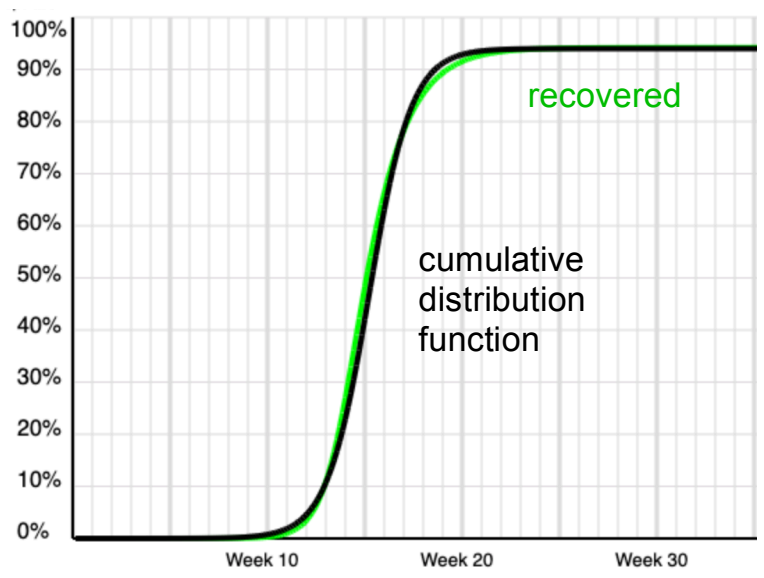

(b) The cumulative distribution S curve fits the SIR model

Figure 2. An example of the SIR model.

Although the SIR model gives a good understanding of the shape of epidemics and explains why vaccination works (the number of susceptible people in a population decreases so that the 
infection cannot reach exponential growth) it has limited value for forecasting because externally induced changes are likely. For example, it cannot support an analysis of the WHO's test-trace-isolate policy which, in the absence of a vaccine, is seen by many as the way to control a pandemic.

Figure 2(b) shows the black $S$ curve of the cumulative distribution function $f(x)=1 /\left(1+e^{-(x-\mu) / s}\right)$ is a good fit to the green curve of recovered people generated by the Kermack-McKendrick model. Related S curves occur in other well-known applications such as the Bass Diffusion model [13], a differential equation for the S-curve, which is a universal feature of adoption and spread of technology in populations. The Bass model can forecast the total number of adoptions and the inflection point of the S-curve. Using calibrated curves, such as the S-curve models, works well in diffusion models when there is no change in the underlying dynamics. Social distancing or lockdowns changes the underlying dynamics and makes these models inappropriate for forecasting.

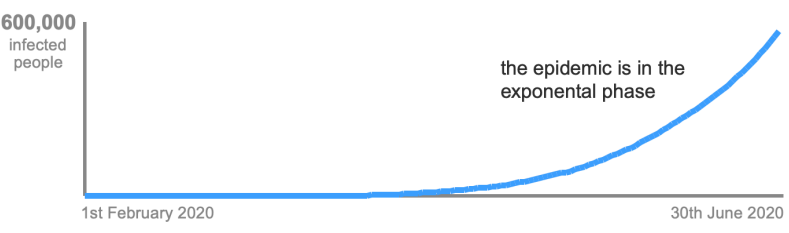

(a) numbers of people infected in India

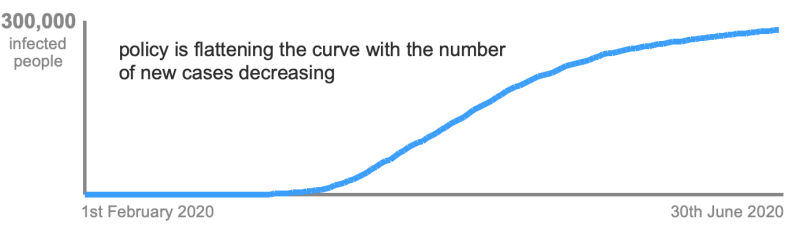

(c) numbers of people infected in the UK

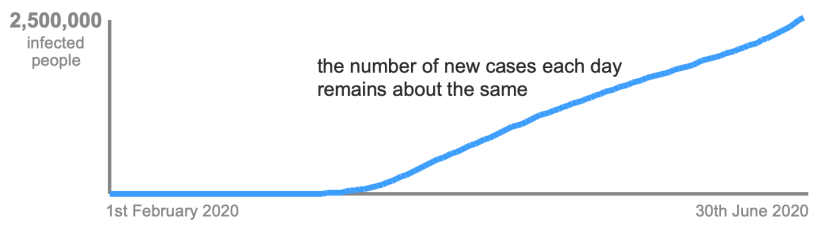

(b) numbers of people infected in the USA

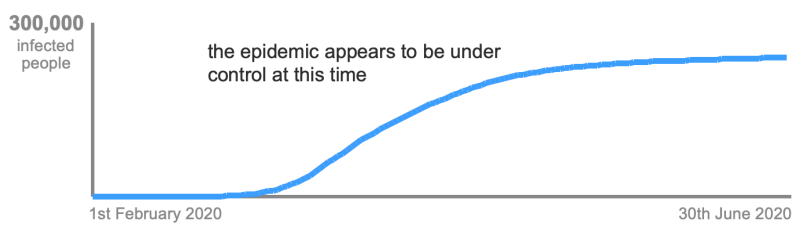

(d) numbers of people infected in Italy

Figure 3. The phases of the empirical curves do not fit the $S$ model.

Two major problems make forecasting epidemics difficult: changes in social behavior altering the underlying dynamics and the availability of reliable data.

The first is illustrated by the different shaped curved in Figure 3. On June 30th India was still in the exponential phase of the S curve. However after March 28th the USA no longer had an $S$ curve but had an almost linear trajectory. In contrast the number of new infections in the U.K. tails off in June while in Italy the total number of people newly infected tails off towards zero. The reason these curves deviate from the $S$ curve is that policy and changes in public behavior have changed the reproduction number. Although policy makers hope their policies will workand these curves give retrospective evidence that some policies have worked-modeling by curve fitting has limited value for forecasting when there is no way of knowing when one kind of curve will transform into another. For example, in September 2020 many countries have 
experienced new exponential growth and are now preparing for a "second wave" not evidenced in the graphs of Figure 3.

The second problem with these models is that the data in many countries can be very poor. For example in the U.K. there is systemic underreporting at weekends as new cases wait to be added to the statistics for Monday or Tuesday. Worse is that infections may be missed or misclassified. Even worse is in the absence of comprehensive testing we do not know how many people have had the virus, how many people are asymptomatic, and how many people are naturally immune. Thus the proportion of susceptible people in the population may be much lower than 100 percent with a significant impact on the model. Even the best models cannot give meaningful forecasts in the absence of reliable data.

In epidemiology with a new disease, such as COVID-19, there is no way to validate the model. The best that can be done is taking models from similar past diseases and modifying them according to your assumptions for future parameters. This is much more challenging than standard scientific modeling because the model and the parameter forecast methods cannot be validated beforehand.

Parameter forecasting for the Kermack-McKendrick model is a particular difficulty. For example initial estimates of the initial reproduction for COVID-19, $R_{0}$, were in the range 1.9 to 5.7 [14]. This makes a huge difference to the simulated evolution of an epidemic.

\section{Agent-Based Modeling}

One of the major tools of complex systems science is agent based modeling for computer simulation. Instead of trying to model macro-level statistics such as the number of people infected in a nation, in this approach these numbers emerge from the micro-level interactions of simulated agents (people in this case). In the simplest cases people are represented by colored dots on a computer screen: red for infected, blue for susceptible, and grey for recovered. The dots move around the screen and when a red infected dot gets close to a blue uninfected susceptible dot, with a given probability the susceptible agent becomes infected. This is illustrated by the sequence in Figure 4. At the beginning (top left) no agents are infected, at the top right the infection is in the exponential phase, at the bottom left the red peak of the infection has been passed, and at the bottom right the epidemic is almost over. 

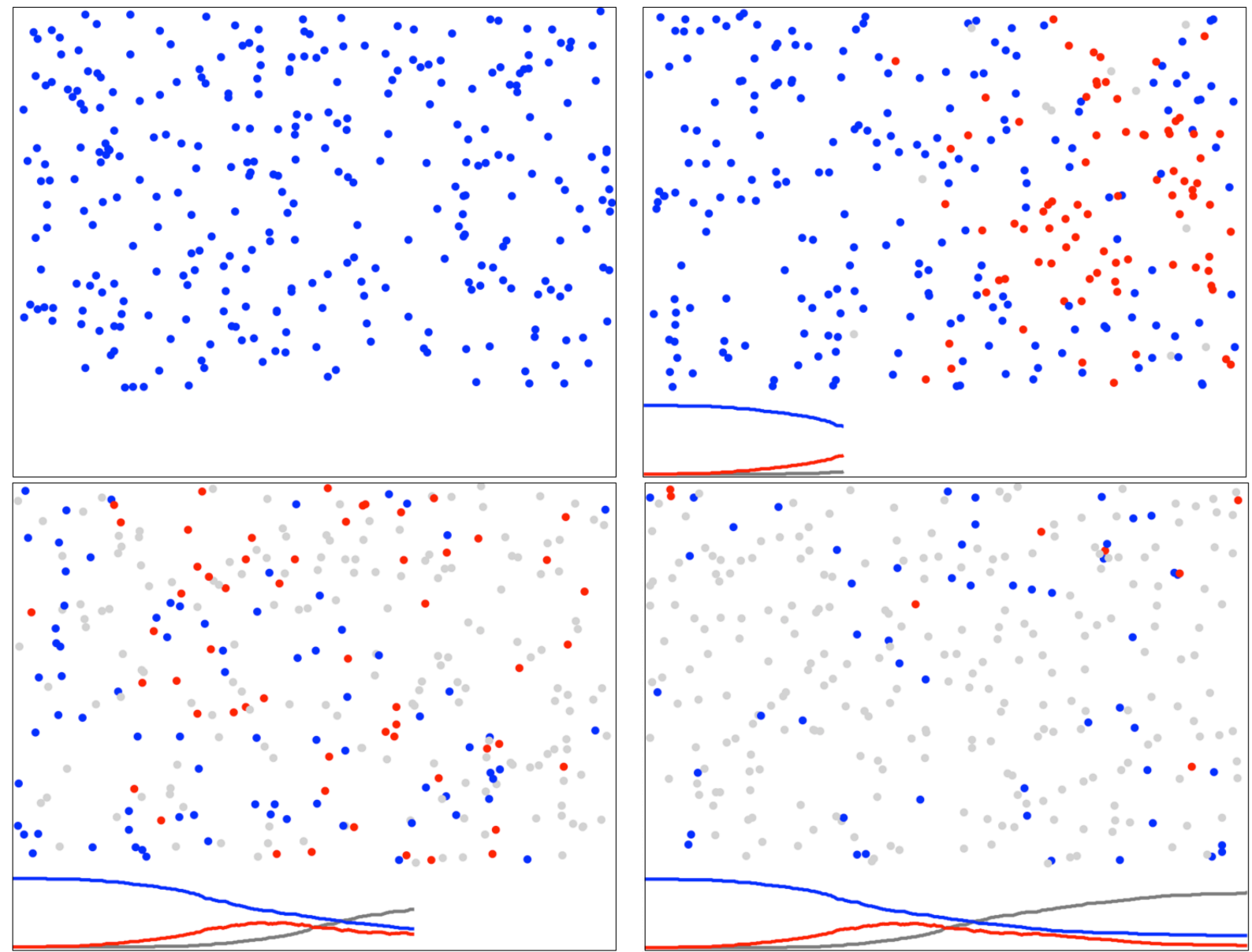

Figure 4. An agent-based simulation of an epidemic.

A model like this is makes it easy to understand the epidemic process and the lay person can see the exponential growth of the epidemic and how epidemics can jump from one country to another. However, although these animations can be alluring they depend on the assumptions built into the simulation. Lay people may be willing to trust that the modelers' assumptions are reasonable, but they have no way check. Modelers do not always present the results of sensitivity analyses (small changes in modeling assumptions), making it harder for lay people to be skeptical and question a model's output.

In the absence of a vaccine, as lockdown is eased the number of susceptible people will remain high (although without adequate testing exactly how many cannot be known). This means there could be sufficient susceptible people in the population for the epidemic to re-emerge, possibly requiring another even more socially and economically damaging lockdown. In many countries the epidemic is being controlled by the well-established test-trace-isolate procedures of epidemiologists. Computer modeling has enabled this policy to be tested in silico and the results suggest it will be very successful-as it has been in the countries than have implemented it. However some countries do not have effective testing and this makes controlling the epidemic more difficult. 


\section{Are the Models Wrong and Dangerous?}

Here is where the controversies exist. A modeler might say the parameters are based on real data (evidence based) but that does not overcome questionable assumptions. Some policy makers are challenging the models because they are not sure the right "evidence" is being used or the assumptions are right. Other policy makers are making value judgments about tradeoffs between what the models say and the social and economic costs.

Alex Berenson gives a scathing account of the Imperial College model that led to the lockdown policy in the U.K. and the University of Washington Model that has informed policy makers in the USA. In his view these models are indeed wrong and dangerous [15]. Sharon Begly also criticizes the Washington University model, which she says is flawed and should not be used to guide U.S. policies [16]. Certainly some models proposed by scientists are contradicted by the available evidence, and some arguments made by scientists are flawed logically, empirically, or both. Modern science can be big business and human weaknesses can overcome scientific integrity. Consulting for the government can fund laboratories and make successful careers, but it can also compromise objectivity.

\section{Computational Modeling and Future COVID-19 Policies}

Democracy has been described as the least worst form of government. In a similar vein, computational modeling could be described as the least worst way of anticipating the future for policy purposes.

At the time of this writing (September 2020) citizens and business in many countries are desperate to end lockdown and governments are responding. In the U.K. the 2-meter separation guidance has been replaced by a 1-meter rule suggesting that political pressure may override evidence-based modeling. It appears politicians are diverging from their scientific advisors and making decisions to relax the lockdown on political as well as scientific grounds. However some question whether England began ending its lockdown in July too soon [17]. It remains to be seen if the risks taken for political reasons pay off, or if they release new waves of infection in the great majority of the U.K. population believed to be uninfected and susceptible. The stakes are high. In August 2020 the USA and the U.K saw new epidemic hotspots emerge and it is clear that the pandemic is not close to being over, as the World Health Organization warns. In September 2020 the British Prime Minister said the U.K. is "now seeing a second wave" of COVID-19 and that "It's been inevitable we'd see it in this country" [18].

There are many subtle patterns of infection dynamics that could still occur, including transmission on "star networks" as local towns and cities become infected. How can we know whether or not this will happen? Without modeling we go into the unknown. With modelingassuming transparency about its methods, data, and assumptions-we have a vision of possible 
futures that can help us take the safest paths with the least risk and best chance of good outcomes.

Managing the pandemic on the ground is very complicated. Although we have good models of how COVID-19 is spread the number of variations for any particular case can be large. For example, consider children returning to school. The interactions between children can be very varied as they move through corridors between classrooms, interact in class, and travel to and from school. Even though most schools have their own individual layouts it would be possible to build computer models and simulate movements and infections starting from many sets of initial conditions. Similarly, simulations of movements and interactions could reassure workers that it is safe to go back to their city center office or inform managers that further measures are required to make the workplace safe. Such models let us run "what if" scenarios that reveal possible futures based on a given set of assumptions. Policy makers have to decide which scenarios to take more seriously and implement policies that will maximize the chance of the desired future actually occurring. The use of models to run scenarios is not the same as running them to make predictions.

\section{Political Leadership}

In the end good policies result from good leadership. The leaders decide what to do and use the models for advice. This is what Winston Churchill was driving at when he said, "scientists should be on tap, not on top."

When the pandemic unfolded many leaders were criticized for lack of preparedness. But governments find it constitutionally challenging to spend resources on possible events that seem remote and unlikely - there are so many urgent things to do in the present. The public will support attending to the urgent but not preparing for the rainy day. Some scientists were warning that pandemic danger was getting high because of international travel and the increasing number of labs experimenting with genetically modified viruses. For example, in 2015 Bill Gates gave a TED talk warning of an imminent pandemic [19]. But such warnings could gather no following and few governments were prepared. The ones that did the best (Taiwan and South Korea) had already had previous close brushes with the SARS coronavirus and took steps to be prepared for another surge. It is likely that governments now have the public support they need to prepare for future pandemics. This preparation can be informed by computer modeling.

\section{Summary}

Computational modeling is an essential input to policy for managing major disruptive events such as the COVID-19 pandemic. Two major approaches to modeling have been presented: curve fitting to macro-level models based on differential equations and agent-based modeling 
based on interactions at the micro level. The former are aggregate macro-level numerical models while the latter disaggregate micro-level models based on hypotheses of agent behavior within their environment. While politicians like very much the simplicity of $\mathrm{R}$ to make policy arguments, experts warn about important weaknesses of calculating/estimating of this number at the country and regional level [20].

Macro-level models are useful for providing forecasts of future numbers. Agent-based models go further because they give insights into the space of future possibilities. The macro-level models are susceptible to error due to externally induced phase change-for example, lockdown completely changes the shapes of the curves. Agent-based models can model phase changes yielding a better insights into the possible future. All these models can give poor results, especially when the data are poor.

There is an inevitable tension between politicians and scientists since the former want simple single answers to their questions, while the latter present many alternatives reflecting the way that science works. Science does not present a single view but has many competing ways to model new phenomena. The argument "we follow the science" is undermined when the scientists can't agree among themselves.

We believe computational models are the best we have to guide policy. Since models can give bad forecasts, modelers should make their assumption clear (including data assumptions) so that others can decide. Ultimately policy makers must make decisions, including which models to accept or reject within a wider political context. Furthermore, policy decisions must take into consideration a delicate trade off between public health and the economic and social damage of pandemic catastrophe leading to deep recession and social unrest.

Computational modeling will be important in the recovery from the pandemic over the next few years for meta modeling pandemics waves, economic impact, and social change. These are highly intertwined and immensely complex problems. They are singular, history-making events deserving better attention and the deeper understanding that computational modeling can provide.

\section{Further information}

More detail can be found in the free online course 'COVID-19: Pandemics, Modelling, and Policy produced by the UNESCO UniTwin Complex Systems Digital Campus. 


\section{References}

[1] Walker, P.G.T., Whittaker, C., Watson, O. et al. The global impact of COVID-19 and strategies for mitigation and suppression. Imperial College London (March 26, 2020). DOI: https://doi.org/10.25561/77735.

[2] WHO Director-General's opening remarks at the media briefing on COVID-19 - 11 March 2020. World Health Organization, March 11, 2020.

[3] World Health Authority 'Coronavirus disease 2019 (COVID-19), Situation Report-73. World Health Organization. April 2, 2020.

[4] Horowitz, J. Italy's health care system groans under coronavirus a warning to the world. New York Times. March 12, 2020.

[5] House of Commons Statement by The Secretary of State for Health and Social Care. Hansard, Volume 673. U.K. Parliament. March 11, 2020.

[6] Stone, J. Top scientists set up 'shadow' SAGE committee to advise government amid concerns over political interference The Independent Newspaper. May 3, 2020.

[7] BBC News. Coronavirus: U.K. firms slash more than 12,000 jobs in two days. July 1, 2020,

[8] BBC News. Coronavirus: U.K. failed to stockpile crucial PPE. April 28, 2020.

[9] Solé, R. et al. Phase transitions and complex systems. Complexity 1 (1996), 13-26.

[10] San Miguel, M, Johnson, J. H., Kertesz, J., Kaski, K., Díaz-Guilera, A., MacKay, R. S., Loreto, V., Érdi, P., and Helbing, D. Challenges in complex systems science. The European Physical Journal Special Topics 214 (2012), 245-271.

[11] Kermack, W. O. and McKendrick, A. G.A contribution to the mathematical theory of epidemics. Proc. R. Soc. A. 115, 772 (1927).

[12] Anderson, R. M. and May, R. M. Infectious Diseases Of Humans: Dynamics and control. Oxford University Press, 1992.

[13] Mahajan, V., Muller, E., and Bass, F. M. Diffusion of new products: empirical generalizations and managerial uses. Marketing Science 14, 3 (1995).

[14] FutureLearn. Pandemics, Modelling, and Policy, Step 1.7. The Kermack-McKendrick SIR epidemic model. April 2020.

[15] Berenson, A. The failure of expert predictions and models. Hillsdale College. April 28, 2020

[16] Begly, S. Influential COVID-19 model uses flawed methods and shouldn't guide U.S. policies, critics say. STAT. April 17, 2020.

[17] Is England ending its lockdown too soon? The Economist. June 4, 2020.

[18] BBC News. COVID: U.K. seeing second wave, says Boris Johnson. September 18, 2020

[19] Gates, B. The next outbreak. We're not ready. TED Talk. March 15, 2015.

[20] Adam, D. The Limits of R. Nature 583, 7816 (2020), 346-348.

\section{Biographies}

Jeffrey Johnson is Professor of Complexity Science and Design at the Open University in the U.K., Deputy President of the UNESCO UniTwin Complex Systems Digital Campus and PastPresident of the Complex Systems Society. His research interests are in the dynamics of 
complex multilevel social and environmental systems, and in systems thinking and computational complex systems science in policy and management. He has many years experience of online education. He is an associate editor of ACM Ubiquity.

Peter J. Denning is past president of ACM (1980-82) and is Distinguished Professor, Chair of the Computer Science Department, and Director of the Cebrowski Institute at the Naval Postgraduate School in Monterey, California.

Kemal Delic is a senior visiting research fellow at the Center for Complexity and Design at the Open University. He is the co-founder of Al-Inc, Ltd. and a lecturer at the University of Grenoble and University of Sarajevo. He is advisor and expert evaluator to the European Commission. He previously held positions as a senior enterprise architect and senior technologist and scientist at Hewlett-Packard.

Dr. Jane Bromley is lecturer in Computing and Cyber Security at the Open University. Her research is at the interface of $\mathrm{Al}$ and psychology, including how we learn and make machines that learn. Her current project investigates how humans carry out difficult image classification tasks and how machines can be made to do this.

DOI: $10.1145 / 3427634$ 